\title{
Nanocrystalline cellulose decorated quantum dots based tyrosinase biosensor for phenol determination
}

\begin{abstract}
Novel biosensor architecture based on nanocrystalline cellulose (NCC)/CdS quantum dots (QDs) nanocomposite was developed for phenol determination. This nanocomposite was prepared with slight modification of nanocrystalline cellulose (NCC) with cationic surfactant of cetyltriammonium bromide $(\mathrm{CTAB})$ and further decorated with 3-mercaptopropionic acid (3-MPA) capped CdS QDs. The nanocomposite material was then employed as scaffold for immobilization of tyrosinase enzyme (Tyr). The electrocatalytic response of Tyr/CTABNCC/QDs nanocomposite towards phenol was evaluated using differential pulse voltammetry (DPV). The current response obtained is proportional to the concentration of phenol which attributed to the reduction of o-quinone produced at the surface of the modified electrode. Under the optimal conditions, the biosensor exhibits good linearity towards phenol in the concentration range of 5-40 $\mu \mathrm{M}(\mathrm{R} 2=0.9904)$ with sensitivity and limit of detection (LOD) of $0.078 \mu \mathrm{A} / \mu \mathrm{M}$ and $0.082 \mu \mathrm{M}$, respectively.
\end{abstract}

Keyword: Nanocrystalline cellulose; Biosensor; Tyrosinase; Electrochemical; Quantum dots 